\title{
MRI in active surveillance: a critical review
}

Vasilis Stavrinides $^{1}$, Francesco Giganti ${ }^{1,2}$, Mark Emberton ${ }^{1,3}$, Caroline M Moore ${ }^{1,3}$.

\section{Affiliation addresses:}

${ }^{1}$ Division of Surgery and Interventional Science, University College London, Charles Bell House, 43-45 Foley Street, W1W 7TS, London, UK

2 Department of Radiology, University College London Hospitals NHS Trust, 235 Euston Road, NW1 2BU, London, UK

${ }^{3}$ Department of Urology, University College London Hospitals NHS Trust, 235 Euston Road, NW1 2BU, London, UK

Word count: 3504 (including abstract)

\section{Corresponding author:}

Vasilis Stavrinides

Division of Surgery and Interventional Science, University College London, Charles Bell House, 43-45 Foley Street, W1W 7TS, London, UK 


\section{Abstract}

Introduction: Recent technological advancements and the introduction of modern anatomical and functional sequences have led to a growing role for multiparametric magnetic resonance imaging ( $\mathrm{mpMRI})$ in the detection, risk assessment and monitoring of early prostate cancer. This includes men who have been diagnosed with lower risk prostate cancer and are looking at the option of active surveillance (AS). The purpose of this paper is to review the recent evidence supporting the use of mpMRI at different time points in AS, as well as to discuss some of its potential pitfalls.

Methods: A combination of electronic and manual searching methods were used to identify recent, important papers investigating the role of $\mathrm{mpMRI}$ in $\mathrm{AS}$.

Results: The high negative predictive value of $\mathrm{mpMRI}$ can be exploited for the selection of AS candidates. In addition, mpMRI can be efficiently used to detect higher risk disease in patients already on surveillance.

Conclusion: Although there is an ongoing debate regarding the precise nature of its optimal implementation, mpMRI is a promising risk stratification tool and should be considered for men on AS. 


\section{Introduction}

Active surveillance (AS) for early prostate cancer is an alternative to radical surgery or radiotherapy that emerged after the growing awareness that many cancers diagnosed through prostate-specific antigen (PSA) screening are low-risk and unlikely to metastasize(1-3). AS is directed towards men with low or intermediate risk disease with a life expectancy of over 10 years and requires that both clinician and patient assess and balance the risk of the proven cancer against the potential side effects of radical treatments. There currently are many AS programs across the world with excellent outcomes in terms of overall and prostate cancer specific mortality(4-8). Although their exact selection criteria differ, they all use variations of the D'Amico or National Comprehensive Cancer Network (NCCN) classifications to identify low-risk disease (low-risk: PSA $\leq 10 \mathrm{ng} / \mathrm{mL}$, Gleason score $\leq 6$, stage T1-T2a; intermediate risk: $10<\mathrm{PSA}<20 \mathrm{ng} / \mathrm{mL}$, Gleason score 7, stage T2b; highrisk: PSA >20 ng/mL, Gleason score $\geq 8$, stage T2c-T3a)(9,10). Patients undergo regular clinical examinations, PSA testing and prostate biopsy, while progression is defined using pre-established clinical or pathological thresholds for clinical significance. In the AS context only "clinically significant" cancers are treated and "progression" implies to a shift from "insignificant" to "significant" disease with potential for adverse consequences if the cancer remained untreated.

Multiparametric magnetic resonance imaging (mpMRI) has shown considerable potential as a risk stratification tool and is likely to be incorporated more widely into patient pathways in coming years(11). The use of imaging parameters to include or exclude men, or to routinely reassess men on active surveillance varies between different programmes, with a lack of published data on the precise timing of imaging, or of thresholds which should prompt additional biopsy or a move to active treatment. The UK National Institute for Health 
\& Care Excellence is the first national body to recommend that $\mathrm{MRI}$ is used in men considering active surveillance, and that it can also be used instead of, or in addition to biopsy during follow up(12).

\section{Multiparametric MRI (mpMRI)}

MpMRI consists of a combination of T2-weighted (T2W), diffusion-weighted (DW) and dynamic contrast-enhanced (DCE) sequences. There are a number of studies attempting to measure the ability of $\mathrm{mpMRI}$ to diagnose significant cancer against a reference standard, usually either a needle biopsy interrogation of the prostate or pathological specimens obtained after radical prostatectomy (RP). On the whole, such studies have demonstrated that mpMRI (i) has a high sensitivity and negative predictive value $(13,14)$ (ii) misses mostly small, low-grade tumours $(15,16)$ (iii) is associated with clinically significant disease when positive(17) and (iv) allows estimation of disease burden at diagnosis. The recently published PRECISION study has shown within a randomised controlled trial design that when an mpMRI-targeted and standard biopsy approach are compared, there is greater detection of clinically significant disease, less detection of clinically insignificant disease and fewer men undergo biopsy(18). These attributes are also appealing in the setting of active surveillance where it is known that a man has lower risk disease and the aim is to detect only that cancer which is more likely to benefit from treatment.

MpMRI reporting systems standardize the diagnostic process so that results are easier to analyze, reproduce and communicate. The Likert scale is a 5-point ordinal scale used to assign a likelihood of clinically significant cancer based on mpMRI appearances of the lesion and the background of the whole prostate and higher Likert scores (i.e. 4 or 5 ) are associated with increased significant cancer detection(19). Although this scoring system 
has been applied in important studies such as the PROMIS trial(13), it implies a subjective assessment of the whole prostate gland (for example ruling in or out lesions when there is a background of inflammation) and is highly influenced by the expertise of the reporting radiologist. A more standardised 5-point scoring system (Prostate Imaging Reporting and Data System [PI-RADS]) for prostate lesions was introduced in 2012(20) and then updated to version 2.0 in 2014(21). In summary, according to this system, the dominant sequence for detecting cancer in the peripheral zone is DWI, whereas for the transition zone this is T2WI. For DWI, apparent diffusion coefficients (ADC) and high b-values $\left(>1400 \mathrm{~s} / \mathrm{mm}^{2}\right)$ are mandatory, whereas DCE has a secondary role (only the presence or absence of focal enhancement is evaluated). Up to four lesions of category 3,4 and 5 are allocated on a sector map and the index lesion is also identified.

\section{MRI for the selection of AS candidates}

\section{Evidence from mpMRI prior to additional biopsy studies in men on AS}

Studies indicate that mpMRI can be very useful for selecting AS candidates(2231)(Table 1). Early work on 85 men fulfilling the Johns Hopkins criteria for AS on standard transrectal biopsy (PSA density $\leq 0.15 \mathrm{ng} / \mathrm{mL} / \mathrm{cc}, \leq 2$ positive cores, $\leq 50 \%$ tumour in any core, Gleason score $\leq 6$, and stage T1c) who had an additional mpMRI and 12-zone-plustargeted TRUS biopsy concluded that $29 \%$ of patients did not meet AS eligibility criteria(32). MpMRI lesion number and degree of suspicion were associated with pathological reclassification. Similar work has demonstrated that, in men previously diagnosed using a standard systematic approach, mpMRI-plus-targeted biopsy often reclassifies patients as AS-ineligible, with reclassification rates ranging from $10 \%$ to as high as $59 \%(22,23)$. High- 
suspicion lesions on mpMRI (e.g. PIRADS v2 4-5) are strongly associated with underlying clinically significant disease and higher reclassification rates(26,28,29). In contrast, evidence suggests that low mpMRI suspicion scores are not associated with pathological upgrading(30). It has also been shown that clinical decision tools incorporating mpMRI scores can predict disqualification from AS and could, therefore, have utility when counseling men considering AS(27). Abdi and colleagues assessed whether mpMRI combined with mpMRI fusion technology during TRUS biopsy can enhance the detection of significant disease in 111 men on AS, using termination of surveillance as the primary end point(24). MpMRI increased the rate of AS termination where PI-RADS score of 4 or 5 (versus $\leq 3$ ) was the only significant predictor, essentially meaning that patients with a suspicious lesion on mpMRI are more likely to require active treatment.

Guo and colleagues synthesized findings on mpMRI performance for predicting upgrading on confirmatory biopsy by conducting a meta-analysis on diagnostic data from 1028 patients(33). The pooled sensitivity, specificity, positive likelihood ratio and negative likelihood ratio estimates for re-classification were $0.69,0.78,3.1$ and 0.4 , respectively. This further reinforces the view that although a negative MRI in an AS candidate is reassuring, an MRI lesion should be targeted at biopsy due to the higher risk of harbouring clinically significant disease.

\section{Evidence from radical prostatectomy studies in men potentially suitable for AS}

Despite some early reports that mpMRI cannot predict upgrading, upstaging or positive surgical margins at RP in otherwise AS-eligible men(34), there is a good body of evidence suggesting the contrary(35-37) (Table 1). De Cobelli and colleagues reviewed 2200 men who had a previous RP and isolated 223 patients that fulfilled the Prostate 
Cancer Research International Active Surveillance (PRIAS) criteria for surveillance (PSA level $<10 \mathrm{ng} / \mathrm{mL}$ at diagnosis, PSA density $<0.2 \mathrm{ng} / \mathrm{mL} / \mathrm{cc}$, clinical stage T1c or T2, Gleason score $\leq 3+3$, and one or two biopsy-positive cores)(35). These men had 1.5T mpMRI 6-8 weeks after TRUS biopsy. Out of 113 patients who experienced upgrading, 112 had MRIvisible cancer and only one did not and the estimated sensitivity of mpMRI for extracapsular extension was $100 \%$.

Tay and colleagues retrospectively assessed 208 men who underwent RP after mpMRI and classified them for AS suitability according to three different AS criteria, i.e. Epstein (T1c, PSA $\leq 10 \mathrm{ng} / \mathrm{mL}$, Gleason $\leq 6, P S A D \leq 0.15 \mathrm{ng} / \mathrm{mL} / \mathrm{cc}, \leq 2$ positive cores and core involvement $\leq 50 \%$ ) and low-risk or extended NCCN criteria (low-risk: Gleason $\leq 3+3 / P G G ~ 2$, Stage $\leq T 2 a, P S A \leq 10 \mathrm{ng} / \mathrm{mL}$; extended: Gleason $\leq 3+4 / P G G 1$, Stage $\leq T 2 b$, PSA $\leq 15 \mathrm{ng} / \mathrm{mL}$ )(37). Combining mpMRI with NCCN low-risk clinical criteria resulted in a statistically significant improvement in specificity $(0.8,95 \% \mathrm{Cl}: 0.28-0.99)$ and area under the curve $(0.9,95 \% \mathrm{Cl}: 0.70-1.00)$ for selecting AS candidates. A retrospective study of 126 patients who were eligible for AS but treated with RP by Porpiglia and colleagues revealed that incorporating mpMRI to multivariate models including the PRIAS (T1c or T2, Gleason $\leq 6, \leq 2$ positive cores, PSA $\leq 10 \mathrm{ng} / \mathrm{mL}, \mathrm{PSA}$ density $\leq 0.2 \mathrm{ng} / \mathrm{mL} / \mathrm{cc}$ ) and Epstein AS criteria increased their accuracy for predicting pathologically confirmed insignificant prostate cancer by $5 \%$ and $7 \%$, respectively(36). In the same study, the sensitivity of mpMRI for detecting significant cancer was $73.7 \%$, specificity $60.8 \%$, PPV $60.8 \%$ and NPV $73.7 \%$, in line with other similar studies (Figure 1).

\section{Repeat mpMRI as a tool to detect change in men on surveillance}


MpMRI is also of interest in detecting change in men on AS and helping patients with stable imaging findings to avoid routine re-biopsy. The optimal imaging schedule to balance the costs and benefits of this approach is not well defined, and is likely to differ with baseline risk, such that men with either Gleason $3+4$ disease or a visible lesion are more likely to show radiological change over time.

In an attempt to define the natural history of small mpMRI lesions, Rais-Bahrami and colleagues retrospectively reviewed 153 patients who underwent a minimum of two mpMRIs followed by mpMRI-TRUS fusion biopsy(38). The growth rate of the lesions was calculated from serial scans, using two index lesion size thresholds (5 and $7 \mathrm{~mm}$ ). The lesions were mostly benign (with only up to $13.8 \%$ harboring low-grade Gleason 6 cancer) and did not change significantly in size over approximately 2 years $(2.31 \pm 1.56$ years and $2.40 \pm 1.77$ years for $\leq 7 \mathrm{~mm}$ and $\leq 5 \mathrm{~mm}$ index lesion thresholds, respectively). From these findings the authors proposed a monitoring interval of at least two years for small lesions.

Studies of men already on AS who have had repeat mpMRls and biopsies are more informative(39-45)(Table 2). In a retrospective study, Walton-Diaz and colleagues evaluated patients meeting surveillance criteria based on systematic TRUS biopsy, who subsequently underwent further systematic biopsies, mpMRI and TRUS targeted biopsies during AS until Gleason grade progression occurred and treatment was recommended(39). The sensitivity, specificity, negative and positive predictive values were 0.53 (95\% Cl: 0.28 0.77), 0.80 (95\% Cl: 0.65-0.91), 0.80 (95\% Cl: 0.65-0.91), 0.53 (95\% Cl: 0.28-0.77), respectively. The authors also concluded that the use of mpMRI decreases the number needed to biopsy in order to detect one pathological progression from 8.75 to 2.89 , thus reducing the biopsy-related morbidity burden on the surveillance population. In another report from the same study group at the Royal Marsden, UK, reduction in apparent diffusion coefficient (ADC) on DWI was associated with pathological progression(46). Specifically, a 
$10 \%$ reduction in tumour ADC indicated progression with sensitivity and specificity of $93 \%$ and $40 \%$, respectively.

In an interesting study by Felker and colleagues, 49 consecutive men on AS with Gleason 6 cancer underwent $\mathrm{mpMRI}$ at baseline and at least six months later, each followed by a targeted prostate biopsy(40). The authors evaluated whether radiological progression (defined as an increase in lesion suspicion score, size or ADC) predicted pathological progression (Gleason 3+4 or higher on subsequent biopsy). The addition of mpMRI-based parameters to models based on clinical variables increased their predictive ability (AUC 0.91 versus 0.87 ). Additional evidence suggests that high mpMRI suspicion scores are directly associated with upgrading, protocol-based treatment change or both(4143).

The question is often asked whether men with a negative mpMRI still need to undergo routine biopsies on surveillance and some authors suggest that this is the case. Hamoen and colleagues found that, in a subset of 111 men on AS who underwent serial mpMRls and MRI-guided biopsies, systematic TRUS biopsy identified a considerable proportion of patients who had a Gleason upgrade to $3+4$ (from 3+3) at one year(44). Thurtle and colleagues assessed men at baseline and follow up with MRI and biopsy, and concluded that at their centre, follow up protocol biopsies identified additional men with Gleason Group 2 or T3 disease compared to mpMRI and targeted biopsy alone(45). However, they only used 2 cores per target, which may have reduced the reliability of the mpMRI-targeted approach. Sanguedolce and colleagues have assessed the features on baseline mpMRI which predict future progression and concluded that both higher lesion volume and higher score are correlated with AS withdrawal and shorter failure-free survival(47).

Detecting disease progression on mpMRI in imaging-based AS cohorts is limited due to a lack of robust data on the natural variability in the appearance of MRI lesions on serial 
mpMRI(48). The PRECISE recommendations were developed by a European School of Oncology task force in order to standardize mpMRI reporting for men who are on AS and undergo regular follow up scans(49). A set of important recommendations for mpMRI reporting was derived through discussion and scores of consensus among experts from a set of 394 statements. As a result, a PRECISE case report form including all important radiological characteristics (e.g. lesion size, assessment of likelihood of clinically significant disease, likelihood of change from previous scan, parameters that changed etc.) was developed in order to facilitate the standardized collection of clinical and imaging data in these cohorts. In addition, a PRECISE checklist for reporting imaging-based AS outcomes includes key information that investigators need to disclose such as imaging, biopsy and clinical attributes. It was not clear to the PRECISE panel which determinant of size was most important, although a recent publication looking at 30 men with an index lesion on MRI suggests that maximum tumour diameter may be associated with the least interobserver reliability(50).

\section{Limitations of mpMRI in active surveillance}

Even though mpMRI is a promising tool, there are limitations that should be considered and not all studies have demonstrated a clear diagnostic superiority of mpMRI compared to existing pathways. Possible reasons are:

(i) False positives. When selecting AS candidates, mpMRI has a relatively low specificity, with false positive results particularly associated with PI-RADS 3 and 4(51). Non-malignant processes such as adenosis, high-grade prostatic intraepithelial neoplasia and post-atrophic hyperplasia within the prostate can mimic well-differentiated cancer(52). In addition, technical pitfalls (such as improperly 
positioned endorectal coils or patient movement), iatrogenic causes (e.g. post biopsy changes or granulomatous prostatitis post intravesical BCG instillation) and unusual appearance of otherwise normal anatomic structures (e.g. hypertrophic anterior fibromuscular stroma) can lead to a similar result(53). False positive findings lead to interpretation errors and unnecessary biopsies but, nonetheless, care must be taken when the targeted biopsy of a positive mpMRI lesion is negative, as guided biopsies of mpMRI lesions (especially TRUS) can be challenging and should be interpreted in context(54).

(ii) False negatives. Although mpMRI sensitivity increases with tumour size and grade, retrospective studies correlating images with RP specimens suggest that a small proportion of clinically important cancers can be missed or their size underestimated, depending on the quality of the scan and its interpretation(55). It is estimated that the NPV of mpMRI varies considerably from 0.65 to 0.94 and, therefore, a negative mpMRI should be always interpreted in the context of the performance of MRI at that centre(56).

(iii) Variability in infrastructure, image acquisition and interpretation. There appears to be some evidence that PI-RADS/Likert score and the assigned probability of significant cancer vary considerably among radiologists $(57,58)$. Therefore, there is a need for greater clarity of cut-off values for clinically significant disease in different clinical settings(59). Use of the PRECISE guidelines to defining reporting criteria for baseline and subsequent mpMRI will help with the creation of a robustly reported dataset to define radiological thresholds of clinically significant disease in men on AS(49). 


\section{Cost and quality of life considerations}

Many urologists believe that mpMRI could be a useful tool for assessing risk in biopsy-naïve patients and monitoring AS cohorts, but cost and lack of appropriate infrastructure are considered significant obstacles preventing the implementation of imaging-based patient pathways(60). Cost-effectiveness analysis suggests that an MRIguided prostate cancer diagnostic pathway would result in fewer men needing biopsies and greater detection of clinically significant disease, in the prostate cancer detection setting(61). However, uncertainty exists regarding the cost effectiveness of mpMRI specifically in the AS setting, primarily due to the lack of long-term AS data. An Australian study using Markov models and comprehensive sensitivity analyses concluded that the mpMRI-plus-targeted biopsy approach in AS produces fewer biopsies, identifies more significant cancers and diagnoses less insignificant disease, provided that the uptake of AS is increased(62). In the extreme scenario where this strategy was applied to all new cases

of low-risk cancer, the quality of life gain was consistently better while the likelihood of mpMRI being cost-effective was $86.9 \%$. In conclusion, although mpMRI appears to improve quality of life and cost efficiency, more transparent, robust studies clearly focusing on the AS setting and taking into account the economic particularities of different healthcare systems are needed.

\section{Guidelines}


The European Association of Urology (EAU) guidelines strongly recommend mpMRI prior to a confirmatory biopsy combining both a systematic and targeted approach(63). The American Urological Association advocates that mpMRI should be considered during surveillance, where high quality MRI is available, with this recommendation based on clinical principles or expert opinion(64). The UK National Institute for Clinical Excellence (NICE) released a guideline for the diagnosis and management of prostate cancer in January 2015(12). This advocates early mpMRI assessment of AS candidates at enrolment and with any clinical or PSA change (with or without guided biopsy), although it is less prescriptive in terms of histological or biochemical selection criteria and the actual imaging protocol that should be followed during surveillance(65).

The optimal interval between serial mpMRIs in AS is particularly difficult to define. In an imaging-based AS cohort with a median interval of 24 months (range 4-73 months) between two consecutive mpMRIs, $69 \%$ of patients demonstrated radiological progression strongly associated with protocol-based treatment change(42). In addition, PI-RADS scores of 4 or 5 at baseline accurately predicted Gleason upgrade on subsequent biopsies. It should be noted that, on the whole, studies investigating serial mpMRls overall suffer from a lack of standardization. Different authors use different selection criteria, monitoring protocols, outcomes and reporting methods, making evidence synthesis particularly cumbersome. Large scale, multinational efforts to create a global consensus on AS and the various selection and monitoring criteria (including imaging) could mitigate this limitation(66). At the moment, a reasonable imaging strategy in AS based on available evidence of mpMRI phenotypes and the known natural history of prostate cancer could be $\mathrm{mpMRI}$ at enrolment, 1 year and then every two years. At our institution (University College London Hospitals NHS Trust), mpMRI is performed for all AS patients at baseline and at 1 
year as advocated by NICE. Beyond the first year, mpMRI is repeated at $12-24$ months for men with a visible lesion, and at 24-36 months for men with no visible lesion.

\section{Conclusion}

MpMRI shows great promise in the selection and monitoring of men in AS. It optimizes biopsy procedures, as it reduces the number of cores needed to detect clinically significant cancer and it minimizes the detection of insignificant disease. In addition, its high NPV for the presence of clinically significant tumours (reaching $98 \%$ in some studies) means that a negative scan could be used to confirm eligibility for AS. MpMRI is highly specific for detecting upgrading on confirmatory biopsy (up to $80 \%$ ) and mpMRI-based parameters improve the ability to predict reclassification and upstaging, with an NPV for reclassification often exceeding $90 \%$. However, its sensitivity is somewhat lower in the region of $70 \%$. Finally, mpMRI could facilitate the monitoring of low-risk lesions in AS patients and reduce the need for additional biopsies during surveillance. Adequate training, standardization in imaging protocols and reporting and awareness of the modality's limitations are important to accurately stratify risk in the AS setting.

\section{Acknowledgements}

Francesco Giganti is funded by the University College London (UCL) Graduate Research Scholarship and the Brahm PhD scholarship in memory of Chris Adams. 
Mark Emberton is a United Kingdom National Institute of Health Research (NIHR) Senior Investigator. He receives research support from the UCLH/UCL NIHR Biomedical Research Centre.

Caroline M Moore receives grant funding from Movember, Prostate Cancer UK, the National Institute for Health Research, the EAU Research Foundation and the Medical Research Council.

Conflict of interest: None. 


\section{References}

1. Welch HG, Albertsen PC. Prostate cancer diagnosis and treatment after the introduction of prostate-specific antigen screening: 1986-2005. J Natl Cancer Inst. 2009 Oct 7;101(19):1325-9.

2. Ross HM, Kryvenko ON, Cowan JE, Simko JP, Wheeler TM, Epstein JI. Do adenocarcinomas of the prostate with Gleason score (GS) $\leq 6$ have the potential to metastasize to lymph nodes? Am J Surg Pathol. 2012 Sep;36(9):1346-52.

3. Thompson IM, Pauler DK, Goodman PJ, Tangen CM, Lucia MS, Parnes HL, et al. Prevalence of prostate cancer among men with a prostate-specific antigen level $\leq 4.0$ ng per milliliter. N Engl J Med. 2004;350(22):2239-2246.

4. Welty CJ, Cowan JE, Nguyen H, Shinohara K, Perez N, Greene KL, et al. Extended Followup and Risk Factors for Disease Reclassification in a Large Active Surveillance Cohort for Localized Prostate Cancer. J Urol. 2015 Mar;193(3):807-11.

5. Selvadurai ED, Singhera M, Thomas K, Mohammed K, Woode-Amissah R, Horwich A, et al. Medium-term Outcomes of Active Surveillance for Localised Prostate Cancer. Eur Urol. 2013 Dec;64(6):981-7.

6. Klotz L, Vesprini $D$, Sethukavalan $P$, Jethava $V$, Zhang $L$, Jain $S$, et al. Long-Term Follow-Up of a Large Active Surveillance Cohort of Patients With Prostate Cancer. J Clin Oncol. 2015 Jan 20;33(3):272-7.

7. Tosoian JJ, Mamawala M, Epstein JI, Landis $\mathrm{P}$, Wolf S, Trock BJ, et al. Intermediate and Longer-Term Outcomes From a Prospective Active-Surveillance Program for Favorable-Risk Prostate Cancer. J Clin Oncol. 2015 Oct 20;33(30):3379-85. 
8. Bokhorst LP, Valdagni R, Rannikko A, Kakehi Y, Pickles T, Bangma $\mathrm{CH}$, et al. A Decade of Active Surveillance in the PRIAS Study: An Update and Evaluation of the Criteria Used to Recommend a Switch to Active Treatment. Eur Urol. 2016 Dec;70(6):954-60.

9. Carroll PR, Parsons JK, Andriole G, Bahnson RR, Castle EP, Catalona WJ, et al. NCCN Guidelines Insights: Prostate Cancer Early Detection, Version 2.2016. J Natl Compr Cancer Netw JNCCN. 2016 May;14(5):509-19.

10. D’Amico AV, Whittington R, Malkowicz SB, Schultz D, Blank K, Broderick GA, et al. Biochemical outcome after radical prostatectomy, external beam radiation therapy, or interstitial radiation therapy for clinically localized prostate cancer. JAMA. 1998 Sep $16 ; 280(11): 969-74$.

11. Oberlin DT, Casalino DD, Miller FH, Meeks JJ. Dramatic increase in the utilization of multiparametric magnetic resonance imaging for detection and management of prostate cancer. Abdom Radiol [Internet]. 2016 Nov 17 [cited 2017 Feb 19]; Available from: http://link.springer.com/10.1007/s00261-016-0975-5

12. CG175 NICEGuideline 20151105.pdf.

13. Ahmed HU, El-Shater Bosaily A, Brown LC, Gabe R, Kaplan R, Parmar MK, et al. Diagnostic accuracy of multi-parametric MRI and TRUS biopsy in prostate cancer (PROMIS): a paired validating confirmatory study. The Lancet. 2017 Feb;389(10071):815-22.

14. Chamie K, Sonn GA, Finley DS, Tan N, Margolis DJA, Raman SS, et al. The Role of Magnetic Resonance Imaging in Delineating Clinically Significant Prostate Cancer. Urology. 2014 Feb;83(2):369-75. 
15. Renard-Penna R, Roupret M, Compérat E, Rozet F, Granger B, Barkatz J, et al. Relationship between non-suspicious MRI and insignificant prostate cancer: results from a monocentric study. World J Urol. 2016 May;34(5):673-8.

16. De Visschere PJL, Naesens L, Libbrecht L, Van Praet C, Lumen N, Fonteyne V, et al. What kind of prostate cancers do we miss on multiparametric magnetic resonance imaging? Eur Radiol. 2016 Apr;26(4):1098-107.

17. Weaver JK, Kim EH, Vetter JM, Fowler KJ, Siegel CL, Andriole GL. Presence of Magnetic Resonance Imaging Suspicious Lesion Predicts Gleason 7 or Greater Prostate Cancer in Biopsy-Naive Patients. Urology. 2016 Feb;88:119-24.

18. Kasivisvanathan V, Rannikko AS, Borghi M, Panebianco V, Mynderse LA, Vaarala MH, et al. MRI-Targeted or Standard Biopsy for Prostate-Cancer Diagnosis. N Engl J Med [Internet]. 2018 Mar 18 [cited 2018 Mar 28]; Available from: http://www.nejm.org/doi/10.1056/NEJMoa1801993

19. Costa DN, Lotan Y, Rofsky NM, Roehrborn C, Liu A, Hornberger B, et al. Assessment of Prospectively Assigned Likert Scores for Targeted Magnetic Resonance ImagingTransrectal Ultrasound Fusion Biopsies in Patients with Suspected Prostate Cancer. J Urol. 2016 Jan;195(1):80-7.

20. Barentsz JO, Richenberg J, Clements R, Choyke P, Verma S, Villeirs G, et al. ESUR prostate MR guidelines 2012. Eur Radiol. 2012 Apr;22(4):746-57.

21. Weinreb JC, Barentsz JO, Choyke PL, Cornud F, Haider MA, Macura KJ, et al. PIRADS Prostate Imaging - Reporting and Data System: 2015, Version 2. Eur Urol. 2016 Jan;69(1):16-40. 
22. Marliere F, Puech P, Benkirane A, Villers A, Lemaitre L, Leroy X, et al. The role of MRI-targeted and confirmatory biopsies for cancer upstaging at selection in patients considered for active surveillance for clinically low-risk prostate cancer. World J Urol. 2014 Aug;32(4):951-8.

23. Ouzzane A, Renard-Penna R, Marliere F, Mozer P, Olivier J, Barkatz J, et al. Magnetic Resonance Imaging Targeted Biopsy Improves Selection of Patients Considered for Active Surveillance for Clinically Low Risk Prostate Cancer Based on Systematic Biopsies. J Urol. 2015 Aug;194(2):350-6.

24. Abdi H, Pourmalek F, Zargar H, Walshe T, Harris AC, Chang SD, et al. Multiparametric Magnetic Resonance Imaging Enhances Detection of Significant Tumor in Patients on Active Surveillance for Prostate Cancer. Urology. 2015 Feb;85(2):423-9.

25. Tran GN, Leapman MS, Nguyen HG, Cowan JE, Shinohara K, Westphalen AC, et al. Magnetic Resonance Imaging-Ultrasound Fusion Biopsy During Prostate Cancer Active Surveillance. Eur Urol. 2017 Aug;72(2):275-81.

26. Recabal P, Assel M, Sjoberg DD, Lee D, Laudone VP, Touijer K, et al. The Efficacy of Multiparametric Magnetic Resonance Imaging and Magnetic Resonance Imaging Targeted Biopsy in Risk Classification for Patients with Prostate Cancer on Active Surveillance. J Urol. 2016 Aug;196(2):374-81.

27. Radtke JP, Kuru TH, Bonekamp D, Freitag MT, Wolf MB, Alt CD, et al. Further reduction of disqualification rates by additional MRI-targeted biopsy with transperineal saturation biopsy compared with standard 12-core systematic biopsies for the selection of prostate cancer patients for active surveillance. Prostate Cancer Prostatic Dis. 2016;19(3):283. 
28. Pessoa RR, Viana PC, Mattedi RL, Guglielmetti GB, Cordeiro MD, Coelho RF, et al. Value of 3-Tesla multiparametric magnetic resonance imaging and targeted biopsy for improved risk stratification in patients considered for active surveillance. BJU Int. 2017 Apr;119(4):535-42.

29. Elkjær MC, Andersen MH, Høyer S, Pedersen BG, Borre M. Prostate cancer: in-bore magnetic resonance guided biopsies at active surveillance inclusion improve selection of patients for active treatment. Acta Radiol. 2017 Jul 26;028418511772337.

30. Alberts AR, Roobol MJ, Drost F-JH, van Leenders GJ, Bokhorst LP, Bangma CH, et al. Risk-stratification based on magnetic resonance imaging and prostate-specific antigen density may reduce unnecessary follow-up biopsy procedures in men on active surveillance for low-risk prostate cancer. BJU Int. 2017 Oct;120(4):511-9.

31. Lai WS, Gordetsky JB, Thomas JV, Nix JW, Rais-Bahrami S. Factors predicting prostate cancer upgrading on magnetic resonance imaging-targeted biopsy in an active surveillance population: Nomogram for Prostate Cancer Upgrading. Cancer [Internet]. 2017 Jan [cited 2017 Feb 23]; Available from: http://doi.wiley.com/10.1002/cncr.30548

32. Stamatakis L, Siddiqui MM, Nix JW, Logan J, Rais-Bahrami S, Walton-Diaz A, et al. Accuracy of multiparametric magnetic resonance imaging in confirming eligibility for active surveillance for men with prostate cancer: MRI and Active Surveillance of Prostate Cancer. Cancer. 2013 Sep;119(18):3359-66.

33. Guo R, Cai L, Fan Y, Jin J, Zhou L, Zhang K. Magnetic resonance imaging on disease reclassification among active surveillance candidates with low-risk prostate cancer: a diagnostic meta-analysis. Prostate Cancer Prostatic Dis [Internet]. 2015 [cited 2016 
Jan

http://www.nature.com/pcan/journal/vaop/ncurrent/full/pcan201520a.html

34. Ploussard G, Xylinas E, Durand X, Ouzaïd I, Allory Y, Bouanane M, et al. Magnetic resonance imaging does not improve the prediction of misclassification of prostate cancer patients eligible for active surveillance when the most stringent selection criteria are based on the saturation biopsy scheme: MISCLASSIFICATION OF PROSTATE CANCER PATIENTS ELIGIBLE FOR AS. BJU Int. 2011 Aug;108(4):513-7.

35. de Cobelli O, Terracciano D, Tagliabue E, Raimondi S, Bottero D, Cioffi A, et al. Predicting Pathological Features at Radical Prostatectomy in Patients with Prostate Cancer Eligible for Active Surveillance by Multiparametric Magnetic Resonance Imaging. Bhowmick NA, editor. PLOS ONE. 2015 Oct 7;10(10):e0139696.

36. Porpiglia F, Cantiello F, De Luca S, De Pascale A, Manfredi M, Mele F, et al. Multiparametric magnetic resonance imaging and active surveillance: How to better select insignificant prostate cancer? Int J Urol. 2016 Sep;23(9):752-7.

37. Tay KJ, Gupta RT, Holtz J, Silverman RK, Tsivian E, Schulman A, et al. Does mpMRI improve clinical criteria in selecting men with prostate cancer for active surveillance? Prostate Cancer Prostatic Dis. 2017;20(3):323.

38. Rais-Bahrami S, Turkbey B, Rastinehad AR, Walton-Diaz A, Hoang AN, Siddiqui MM, et al. Natural history of small index lesions suspicious for prostate cancer on multiparametric MRI: recommendations for interval imaging follow-up. Diagn Interv Radiol. 2014 Jul 3;20(4):293-8.

39. Walton Diaz A, Shakir NA, George AK, Rais-Bahrami S, Turkbey B, Rothwax JT, et al. Use of serial multiparametric magnetic resonance imaging in the management of 
patients with prostate cancer on active surveillance. Urol Oncol Semin Orig Investig. 2015 May;33(5):202.e1-202.e7.

40. Felker ER, Wu J, Natarajan S, Margolis DJ, Raman SS, Huang J, et al. Serial Magnetic Resonance Imaging in Active Surveillance of Prostate Cancer: Incremental Value. J Urol. 2016 May;195(5):1421-7.

41. Nassiri N, Margolis DJ, Natarajan S, Sharma DS, Huang J, Dorey FJ, et al. Targeted Biopsy to Detect Gleason Score Upgrading during Active Surveillance for Men with Low versus Intermediate Risk Prostate Cancer. J Urol. 2017 Mar;197(3):632-9.

42. Eineluoto JT, Järvinen $P$, Kenttämies A, Kilpeläinen TP, Vasarainen $\mathrm{H}$, Sandeman $\mathrm{K}$, et al. Repeat multiparametric MRI in prostate cancer patients on active surveillance. PloS One. 2017;12(12):e0189272.

43. Frye TP, George AK, Kilchevsky A, Maruf M, Siddiqui MM, Kongnyuy M, et al. Magnetic Resonance Imaging-Transrectal Ultrasound Guided Fusion Biopsy to Detect Progression in Patients with Existing Lesions on Active Surveillance for Low and Intermediate Risk Prostate Cancer. J Urol. 2017 Mar;197(3):640-6.

44. Hamoen EHJ, Hoeks CMA, Somford DM, van Oort IM, Vergunst H, Oddens JR, et al. Value of Serial Multiparametric Magnetic Resonance Imaging and Magnetic Resonance Imaging-guided Biopsies in Men with Low-risk Prostate Cancer on Active Surveillance After 1 Yr Follow-up. Eur Urol Focus. 2018 Jan 10;

45. Thurtle D, Barrett T, Thankappan-Nair V, Koo B, Warren A, Kastner C, et al. Progression and treatment rates using an active surveillance protocol incorporating image guided baseline biopsies and multi-parametric MRI monitoring for men with 
favourable risk prostate cancer. BJU Int [Internet]. 2018 Feb 13 [cited 2018 Mar 28]; Available from: http://doi.wiley.com/10.1111/bju.14166

46. Morgan VA, Riches SF, Thomas K, Vanas N, Parker C, Giles S, et al. Diffusionweighted magnetic resonance imaging for monitoring prostate cancer progression in patients managed by active surveillance. Br J Radiol. 2011 Jan;84(997):31-7.

47. Sanguedolce F, Petralia G, Sokhi H, Tagliabue E, Anyamene N, Hellawell G, et al. Baseline Multiparametric MRI for Selection of Prostate Cancer Patients Suitable for Active Surveillance: Which Features Matter? Clin Genitourin Cancer [Internet]. 2017 Nov [cited 2018 Mar 28]; Available from: http://linkinghub.elsevier.com/retrieve/pii/S1558767317303361

48. Luckenbaugh AN, Auffenberg GB, Hawken SR, Dhir A, Linsell S, Kaul S, et al. Variation in Guideline Concordant Active Surveillance Followup in Diverse Urology Practices. J Urol. 2017 Mar;197(3):621-6.

49. Moore CM, Giganti F, Albertsen P, Allen C, Bangma C, Briganti A, et al. Reporting Magnetic Resonance Imaging in Men on Active Surveillance for Prostate Cancer: The PRECISE Recommendations-A Report of a European School of Oncology Task Force. Eur Urol [Internet]. 2016 Jun [cited 2016 Jul 18]; Available from: http://linkinghub.elsevier.com/retrieve/pii/S0302283816302810

50. Marin L, Ezziane M, Comperat E, Mozer P, Cancel-Tassin G, Coté J-F, et al. Comparison of semi-automated and manual methods to measure the volume of prostate cancer on magnetic resonance imaging. Diagn Interv Imaging. 2017 May;98(5):423-8. 
51. Mertan FV, Greer MD, Shih JH, George AK, Kongnyuy M, Muthigi A, et al. Prospective Evaluation of the Prostate Imaging Reporting and Data System Version 2 for Prostate Cancer Detection. J Urol. 2016 Sep;196(3):690-6.

52. De Visschere PJL, Vral A, Perletti G, Pattyn E, Praet M, Magri V, et al. Multiparametric magnetic resonance imaging characteristics of normal, benign and malignant conditions in the prostate. Eur Radiol. 2017 May;27(5):2095-109.

53. Panebianco V, Giganti F, Kitzing YX, Cornud F, Campa R, De Rubeis G, et al. An update of pitfalls in prostate mpMRI: a practical approach through the lens of PI-RADS v. 2 guidelines. Insights Imaging. 2018 Feb;9(1):87-101.

54. Quon JS, Moosavi B, Khanna M, Flood TA, Lim CS, Schieda N. False positive and false negative diagnoses of prostate cancer at multi-parametric prostate MRI in active surveillance. Insights Imaging. 2015 Aug;6(4):449-63.

55. Borofsky S, George AK, Gaur S, Bernardo M, Greer MD, Mertan FV, et al. What Are We Missing? False-Negative Cancers at Multiparametric MR Imaging of the Prostate. Radiology. 2018 Jan;286(1):186-95.

56. de Rooij M, Hamoen EHJ, Fütterer JJ, Barentsz JO, Rovers MM. Accuracy of Multiparametric MRI for Prostate Cancer Detection: A Meta-Analysis. Am J Roentgenol. 2014 Feb;202(2):343-51.

57. Sonn GA, Fan RE, Ghanouni P, Wang NN, Brooks JD, Loening AM, et al. Prostate Magnetic Resonance Imaging Interpretation Varies Substantially Across Radiologists. Eur Urol Focus [Internet]. 2017 Dec [cited 2018 May 15]; Available from: http://linkinghub.elsevier.com/retrieve/pii/S2405456917302663 
58. Kasel-Seibert M, Lehmann T, Aschenbach R, Guettler FV, Abubrig M, Grimm M-O, et al. Assessment of PI-RADS v2 for the Detection of Prostate Cancer. Eur J Radiol. 2016 Apr;85(4):726-31.

59. Woo S, Suh CH, Kim SY, Cho JY, Kim SH. Diagnostic Performance of Prostate Imaging Reporting and Data System Version 2 for Detection of Prostate Cancer: A Systematic Review and Diagnostic Meta-analysis. Eur Urol. 2017 Aug;72(2):177-88.

60. Muthigi A, Sidana A, George AK, Kongnyuy M, Maruf M, Valayil S, et al. Current beliefs and practice patterns among urologists regarding prostate magnetic resonance imaging and magnetic resonance-targeted biopsy. Urol Oncol Semin Orig Investig. 2017 Jan;35(1):32.e1-32.e7.

61. Willis SR, van der Meulen J, Valerio M, Miners A, Ahmed HU, Emberton M. A review of economic evaluations of diagnostic strategies using imaging in men at risk of prostate cancer: Curr Opin Urol. 2015 Nov;25(6):483-9.

62. Gordon LG, James R, Tuffaha HW, Lowe A, Yaxley J. Cost-effectiveness analysis of multiparametric MRI with increased active surveillance for low-risk prostate cancer in Australia. J Magn Reson Imaging JMRI. 2017;45(5):1304-15.

63. Prostate Cancer | Uroweb [Internet]. [cited 2018 Jun 4]. Available from: https://uroweb.org/guideline/prostate-cancer/\#6_6

64. American Urological Association - Clinically Localized Prostate Cancer: AUA/ASTRO/SUO Guideline [Internet]. [cited 2018 Jun 4]. Available from: http://www.auanet.org/guidelines/clinically-localized-prostate-cancer-new(aua/astro/suo-guideline-2017) 
65. Streeter EH, Brewster SF, BAUS Section of Oncology. NICE guidelines on Prostate Cancer Active Surveillance: is UK practice leading the world?: Comments. BJU Int. 2015 Jan;115(1):12-3.

66. Bruinsma SM, Zhang L, Roobol MJ, Bangma CH, Steyerberg EW, Nieboer D, et al. The Movember Foundation's GAP3 cohort: a profile of the largest global prostate cancer active surveillance database to date. BJU Int. 2017 Dec 15; 
Table 1: Recent important studies on the value of mpMRI for confirming AS suitability in men with a subsequent biopsy or radical prostatectomy. PRIAS AS criteria: T1c or T2, Gleason $\leq 6, \leq 2$ positive cores, $P S A \leq 10, P S A D \leq 0.2$. Epstein AS criteria: T1c, PSA $\leq 10$, Gleason $\leq 6, P S A D \leq 0.15, \leq 2$ positive cores and core involvement $\leq 50 \%$. NCCN AS criteria (low-risk): Gleason $\leq 3+3 / P G G 1$, Stage $\leq T 2 a, P S A \leq 10$. (TB: targeted biopsy, FB: fusion biopsy, CB: confirmatory biopsy, GS: Gleason score, ECE: extracapsular extension, NCCN: National Comprehensive Cancer Network).

Table 2: Recent important studies on AS patients undergoing mpMRI and repeat biopsy. Low NIH AS criteria: Stage $\leq T 2 a$ PSA $\leq 20$, Gleason $\leq 3+3$; expanded NIH criteria: stage $\leq T 2 a$, PSA $\leq 20$, Gleason $\leq 3+4$, positive cores $\leq 33 \%$. (TB: targeted biopsy, FB: fusion biopsy, SB: systematic biopsy, SN: sensitivity, SP: specificity, PPV: positive predictive value, NPV: negative predictive value, PSAD: PSA density).

Table 3: Take-away summative points for mpMRI in active surveillance.

Figure 1: mpMRI sensitivity and specificity for confirming AS suitability in men with a subsequent biopsy or radical prostatectomy (representative studies with data directly available for analysis). The first four studies use biopsy as a reference standard, whereas the last two use RP. Estimates presented with $95 \%$ confidence intervals (in brackets).

Figure 2: Prostate MRI for AS candidate selection and monitoring. Left panel: Man on active surveillance for a Gleason $3+4,2 \mathrm{~mm}$ prostate cancer on the right side. MRIs at different time points show a right peripheral zone lesion posteriorly, also characterised by low signal intensity on T2WI (A, D, G, J), restricted diffusion ( $B$, $\mathrm{E}, \mathrm{H}, \mathrm{K})$ and focal enhancement $(\mathrm{C}, \mathrm{F}, \mathrm{I}, \mathrm{L})$. The lesion did not show significantly progress and this man is still on AS. Right panel: Man on AS for Gleason 3+4, $8 \mathrm{~mm}$ cancer. The mpMRI scan at baseline (2014) shows a right peripheral zone lesion between the base and midgland, abutting the prostatic capsule and characterised by low signal intensity on T2WI $(A, D)$, restricted diffusion $(B, E)$ and focal enhancement $(C, F)$. In the followup scan (2015), T2W and DCE images suggested progression (increased lesion size and capsular enhancement that may represent early T3a disease). This man underwent radical prostatectomy in 2016, confirming Gleason 3+4 cancer. 